\title{
Increasing the efficiency of local energy markets through residential demand response
}

\author{
Esther Mengelkamp ${ }^{1 *}$ (D), Samrat Bose ${ }^{1,2}$, Enrique Kremers², Jan Eberbach², Bastian Hoffmann² \\ and Christof Weinhardt ${ }^{1}$
}

*Correspondence:

esther.mengelkamp@kit.edu

1 Karlsruhe Institute of Technology,

Fritz-Erler-Str. 23, 76131 Karlsruhe,

Germany

Full list of author information is

available at the end of the article

\begin{abstract}
Local energy markets (LEMs) aim at building up local balances of generation and demand close to real time. A bottom-up energy system made up of several LEMs could reduce energy transmission, renewable curtailment and redispatch measures in the long-term, if managed properly. However, relying on limited local resources, LEMs require flexibility to achieve a high level of self-sufficiency. We introduce demand response (DR) into LEMs as a means of flexibility in residential demand that can be used to increase local self-sufficiency, decrease residual demand power peaks, facilitate local energy balances and reduce the cost of energy supply. We present a simulation study on a 100 household LEM and show how local sufficiency can be increased up to $16 \%$ with local trading and DR. We study three German regulatory scenarios and derive that the electricity price and the annual residual peak demand can be reduced by up to $10 \mathrm{c} € / \mathrm{kWh}$ and $40 \%$.
\end{abstract}

Keywords: Demand response, Local energy market, Reinforcement learning, Agent-based simulation, Peer-to-peer trading

\section{Introduction}

Globally, energy systems are in transition from centrally structured, fossil-fuel based generation towards smaller, less hierarchical and rather decentralized systems based on renewable energy sources (RES) (Gottwalt et al. 2017). This transition requires monetary investments by governments, energy companies, utilities and retailers. In 2011, investments were estimated to reach up to 12.8 trillion USD between 2011 and 2030 for worldwide investments in RES (Bauwens 2016; Intergovernmental Panel on Climate Change (IPCC) 2011). Furthermore, this transition requires a restructuring of generation, transmission and distribution structures.

In this context, the active involvement of end users (e.g. as prosumers) and the exploitation of end user flexibility can pave the way for the creation of local energy balances on a decentralized level (Mengelkamp et al. 2018a; Kamrat 2001). Locally balanced distribution systems lead to a more balanced energy transmission system. This can result in lower transmission congestion and, consequently, reduce the need for redispatch measures and RES curtailment in an energy system. Facilitating a market approach, local energy markets (LEMs) may enable the cost-efficient creation of local energy balances between energy producers, prosumers and consumers. On such LEMs, the participants can trade energy and flexibility among each other.

(c) The Author(s). 2018 Open Access This article is distributed under the terms of the Creative Commons Attribution 4.0 International License (http://creativecommons.org/licenses/by/4.0/), which permits unrestricted use, distribution, and reproduction in any medium, provided you give appropriate credit to the original author(s) and the source, provide a link to the Creative Commons license, and indicate if changes were made. 
Trading in LEMs is currently actively discussed in academic, business and political communities. The focus of interest is on finding a suitable market design (Mengelkamp et al. 2017), privacy issues (Buchmann et al. 2013), coordination of distributed RES (Ampatzis et al. 2014), ancillary services (Rosen and Madlener 2013), and on preferences of residential electricity consumers (Mengelkamp and Weinhardt 2018). Demand response (DR) at the household level (Palensky and Dietrich 2011; Albadi and El-Saadany 2008), remains a fairly new concept that is just beginning to be implemented (e.g. in the FRANKLIN (MWS Projektentwicklungsgesellschaft $\mathrm{mbH}$ 2018) and the SIM4BLOCKS (Hochschule für Technik 2016) projects). The fact that both LEMs and DR in the residential sector are innovative concepts requires a comprehensive and structured approach in order to evaluate the efficiency of their combined application. In this context, we set forth the following research question: How can household energy trading and demand response increase the efficiency of local energy markets?

To answer this question, we set up an agent-based simulation environment consisting of 100 residential households including photovoltaic (PV) and combined heat-and-power (CHP) generation units. This scenario is tailored in size to a typical German low voltage distribution feeder. We choose this size to be eligible for the regulatory advantages introduced in section "Set of scenarios". We introduce a merit order market mechanism for the LEM. The market operates in 15 min trading intervals. We analyze the impact of the degree of interaction among households and DR for three different regulatory scenarios on local self-sufficiency, market closing prices and residual peak demand. We focus on the German context (cf. Energy Industry Act (EnWG) 2005 ${ }^{1}$ ) and use a combination of openly accessible and high quality, self-measured data.

\section{Related work}

\section{Demand response}

All measures that aim at improving the energy system on the consumption side can be grouped together as demand side management (DSM). According to Palensky and Dietrich (2011) these can be divided into the different categories of energy efficiency (EE), time-of-use tariffs (TOU), DR and spinning reserve (SR). This work focuses singularly on DR, instead of all DSM measures. DR includes all changes in consumption behavior over time in response to dynamic energy price changes (Albadi and El-Saadany 2008). It encompasses reductions of energy consumption through curtailment, moving of energy consumption in time and supplementing energy consumption by self-produced energy which reduces the consumption from the grid (Siano 2014). We concentrate on decreasing residential demand and shifting (part) of residential demand to future times.

DR can improve the operation of energy systems based on RES (e.g. curtailing demand in low generation times and shifting it to high generation times) and the planning of RES installation (e.g. reducing the peak demand and, thus, decreasing required installed power capacity). Further, it may decrease the cost of energy consumption (e.g. by shifting part of residential demand towards time intervals with low energy prices) (Albadi and El-Saadany 2008; Pinson and Madsen 2014). Regardless of these projected benefits, the existing market designs seems to hinder the efficient implementation of residential DR in the current German energy system (Cutter et al. 2012; Pinson and Madsen 2014). Nevertheless, the evolution of the smart grid concepts is the first step towards the implementation of DR. Several programs are already underway, that promote DSM measures, 
including DR (Siano 2014). These rely mostly on smart grid information technology, i.e. smart metering infrastructure, to provide the necessary data that enables (residential) consumers to participate in DR (Shariatzadeh et al. 2015). Without the right data and the ability to react to changing prices, DR initiatives cannot be efficiently implemented.

Residential DR has been analyzed in several pilot studies worldwide. One example is a pilot study between 40 Norwegian households, in which a price-based DR mechanism was introduced (Sæle and Grande 2011). The main research focus was on the economic benefits of DR, with a sub-focus on energy consumption reduction. The authors report a positive perception of the pilot project by its participants. Today, real applications of DR in European energy markets seem to be limited to the shifting of larger loads (e.g. heating and cooling) (Prüggler 2013). Apart from individual pilot projects, an overall economic benefit of DR for households seems to only be possible in case that residential RES generation can be sold on a market (Prüggler 2013; Gordijn and Akkermans 2007).

Whereas DR is a growing topic of interest in the smart grid community, the successful implementation of such measures requires specific economic incentives. In section "Local energy markets" we focus on a LEM approach, taking as hypothesis the fact that market mechanisms at a local level improve the integration of DR for residential consumers. Thus, LEMs may provide a bottom-up approach to increase the efficiency of the larger energy system.

\section{Local energy markets}

LEMs support the decentralization of energy systems by including the end users into the energy market (Kamrat 2001; Buchmann et al. 2013; Hvelplund 2006). We define a LEM as a geographically distinct and socially close community of residential prosumers and consumers who may trade locally produced electricity within their community. For this, all actors need to have access to a local market platform on which (buy) bids and (ask) offers for local electricity are matched. LEMs serve different objectives, i.e. strengthening the local economy by keeping financial benefits local, increasing the level of the community's energy autarky, creating incentives to buy energy locally, and empowering energy end users (Koirala et al. 2016; Rosen and Madlener 2013; Mengelkamp et al. 2018a; OlivellaRosell et al. 2018). The most important stakeholders on a LEM are: 1 . Electricity (end) consumers, prosumers and producers, 2. Electricity retailers, 3. Suppliers and generators, 4. Local authorities, and 5. Governments (Kamrat 2001). The enumeration does not represent an ordering of importance. In stakeholder category 1., (end) prosumers and producers related to a small-scale, i.e. residential level, while stakeholder category 3. relates to middle-large scale, e.g. central generation plants. In the long run, LEMs can help to build up a sustainable energy system based on RES by developing local energy balances. Thus, a sustainable energy system that relies on the bottom-up integration of RES can be developed.

The legal and regulatory framework is a key factor for the successful implementation for LEMs. In Germany, the regulatory framework does not specifically support LEMs, yet (cf. the EnWG). Nevertheless, pilot projects of LEMs are being implemented in Germany (e.g. (Mengelkamp et al. 2018)) and worldwide (e.g. (Green and Newman 2017)). However, sustainable business models for LEMs still need to be developed, to ensure their longterm success apart from pilot implementations and proof-of-concepts. 
One of the current main research topics in the LEM context is finding a suitable market mechanism. Several (individual) evaluations of market mechanisms in LEMs exist in form of game theoretic approaches or simulations (Vytelingum et al. 2010; Ilic et al. 2012; Mengelkamp et al. 2017). However, a comprehensive comparison of different market mechanisms and a framework to choose the most appropriate mechanism for a specific LEM use case seem to be missing. To keep the focus on the DR influence on LEM, we use the existing short-term merit order market mechanism of the European Energy Exchange $(E E X)^{2}$ in Germany as a basis (Tveten et al. 2013) and focus on electricity being the sole good traded on the LEM.

Marzband et al. (2013) and Marzband et al. (2014) are two of the few works that already combining a LEM and DR measures. They evaluate an energy management system for a physical microgrid operating in island-mode. They use simulations to analyze the potential cost savings and show how the combination of LEM and DR can reduce overall energy costs. In contrast to Marzband et al. (2013) and Marzband et al. (2014), we rely on individual households as the acting agents in the virtual LEM and only consider the physical implication in terms of the aggregated residual peak demand per year.

\section{Existing modelling approaches}

Existing modelling approaches on (local) energy markets can largely be divided into three categories of: 1. Game-theoretic modelling, 2. Agent-based modelling (ABM) and 3. Case studies. Game-theoretic modelling uses game theory to model, analyze and evaluate the behavior of (local) energy markets according to exogenous and endogenous factors. Singh (1999) define game theory in energy markets as the generalization of decision theory that includes multiple stakeholders and their interaction. It can be divided into non-cooperative (e.g. (Marzband et al. 2016)) and cooperative game theory (e.g. (Jia and Yokoyama 2003)). Game theory mostly strives for finding (Nash) equilibria in their respective models. ABM focuses on the single agent actors and builds a model based on the behavior of single agents and their aggregated behavior (Babic and Podobnik 2016). ABM yields a simulation, that is based on the behavior of simulated, interactive agents and often also yields equilibria in the temporal market behavior. The third approach, case studies, is strictly not a modelling approach, but an implementation of (local) energy markets in reality. Thus, the market is not computationally modelled (simulated), but rather implemented under (fairly) realistic conditions.

In our attempt to include DR in LEMs, we pursue an agent-based simulation approach. The complexity of our LEM with the addition of intelligent adaptive agent strategies and DR would result in a very complex analytic model, which, in contrast to an ABM would require immense computing resources to solve. As we are looking for providing results for several instances and scenarios (see section "Set of scenarios"), we implement a simulation model able to solve additional scenarios and conduct sensitivity analyses within a reasonable time frame. Moreover, ABM allows to include uncertainty and behavioral aspects in the energy system simulation, which allow to capture realistic interactions and overall system behavior in form of emergent phenomena (de Durana et al. 2014; Kremers 2013). ABM has already been used widely for general and electricity market modelling (Sensfuß et al. 2007; Babic and Podobnik 2016). It allows to represent realistic market behavior through a representation of the interactions of the system. In principle, LEMs 
are not different in these aspects to other markets, so an agent-based approach seems reasonable.

There are several academic papers focusing on ABM for DR (e.g. (Rosen and Madlener 2012; Zhou et al. 2011; Yousefi et al. 2011)). However, to the best of our knowledge, there is currently no literature on ABM of DR in combination with LEM as defined in section "Local energy markets". We address this research gap and present a comprehensive $\mathrm{ABM}$ of a LEM with DR and extensive analyses for three regulatory scenarios.

\section{Methodology and model}

\section{Agent definition}

The model represents a LEM with individual residential agents operating on a short-term merit order market in $15 \mathrm{~min}$ time slots. An agent is an autonomous entity which can act proactively inside a common environment and with the ability to interact with the other agents. Our model includes the agent types of prosumers, consumers and producers. Consumer agents are households without electricity generation assets. Prosumer agents are households with electricity generation assets (PV or CHP). They act as producers on the LEM whenever their generation exceeds their demand and as consumers otherwise.

Furthermore, the model requires a market clearing house responsible for LEM trading among the agents. This clearing house facilitates the market mechanism. It collects the bids and offers from the agents and processes them. The clearing house matches bids and offers through a merit order market mechanism. The merit order mechanism results in a uniform market price equal to the highest seller price still being matched.

\section{Model methodology}

Figure 1 shows the overall working of the model. Agents send bid and offers orders to the market clearing house every $15 \mathrm{~min}$ for the next time slot. Once the bids and offers are received by the clearing house, they are sorted in decreasing order for bids and increasing order for offers. Thus, they set up the demand and supply curve that is to be matched through the merit order model. The intersection of the demand and the supply curve determines the market closing price (MCP). It is the uniform price of all accepted transactions. The information about accepted bids and offers is returned back to concerned agents. Once this information is received, the pricing strategy is updated for the next

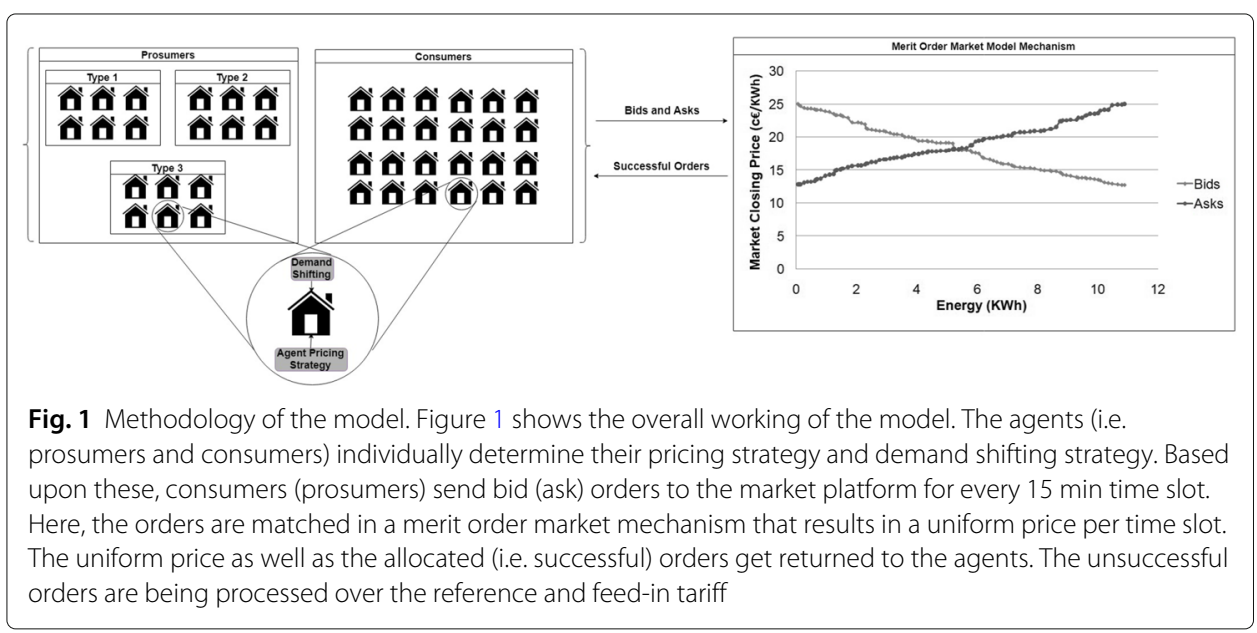


round. The pricing strategy and merit order market mechanism are executed in every time slot. We assume perfect demand and generation forecasts for the next time slot.

The pricing strategy and the demand shifting strategy are executed on an individual local basis for each agent. The pricing strategy of the agents is established through the Erev-Roth algorithm (Erev and Roth 1998; Nicolaisen et al. 2001). It represents the decision making of the agent concerning its pricing. This algorithm promotes those bid and offer prices which previously resulted in high profits and discourages those prices resulting in low profits. The pricing strategy is explained in detail in section "Pricing strategy". The demand shifting strategy is presented in section "Demand shifting for DR".

\section{Pricing strategy}

The agents' main objective is economic rationality (Ilic et al. 2012). Thus, the ultimate goal of all agents is to maximize their profit. Simultaneously, they prefer local energy over grid energy, as long as the local energy is cheaper. Our LEM scenario is embedded in the existing retail energy market. Thus, we consider a reference electricity tariff for residential electricity consumers, and specific feed-in tariffs for PV and CHP producers. Hence, the feed-in tariffs influence the lower market price boundary, while the reference electricity tariff represents the upper boundary. Required taxes, fees and levies also need to be taken into account while calculating the lower market price boundary (cf. Table 1). Agents learn to adapt their bid and offer prices according to the Erev-Roth reinforcement strategy (Erev and Roth 1998; Nicolaisen et al. 2001) within the market price boundaries.

Each agent has a set of strategies $S=\left\{s_{1}, s_{2}, \ldots s_{m}\right\}$ that describes the discrete bid (or offer) prices he can bid to the LEM. This set $S$ contains the integer prices per kWh an agent may bid on the LEM. At the beginning $t_{0}$, agents enter the market without previous knowledge on the LEM's behavior. However, they know the lower $\left(c^{F}\right)$ and upper bound $\left(c^{G}\right)$ of the LEM price. These market bounds represent the grid feed-in tariff $c^{F}$ and the reference tariff $c^{G}$ (see also section "Set of scenarios"). Thus, $S$ contains all prices between $c^{F}$ and $c^{G}$ that agents may bid. Prices are chosen on a discrete $c €$ level with one decimal point. At first, the propensity $q_{i s}(t)$ of agent $i$ to choose strategy $s$ is equal to that of all other strategies. It is set to

$$
q_{i s}\left(t_{0}\right)=\operatorname{sca}\left(t_{0}\right) \frac{\operatorname{avg}\left(\Pi_{i}\left(t_{0}\right)\right)}{|S|} .
$$

with $\operatorname{avg}\left(\Pi_{i}\left(t_{0}\right)\right)$ being the earned profit of an agent in time slot $t_{0}$ and $\operatorname{sca}\left(t_{0}\right)$ being a scaling parameter. Agents in our LEM simulation do not know their average profit yet. Therefore, they need to assume a starting point, e.g. $\operatorname{sca}\left(t_{0}\right) \operatorname{avg}\left(\Pi_{i}\left(t_{0}\right)\right)=1$. Depending on the matched transactions in time slot $t+1$, agents can calculate their profit $\Pi_{i}(t+1)$. Thus, each agent can update the propensity for his strategies in $t+1$ to bid for $t+2$ :

$$
q_{i s}(t+1)=(1-r e c) q_{i s}(t)+\operatorname{MUF}\left(i, s, s^{\prime}, t\right) .
$$

The recency parameter rec determines the weight of impact of the last propensity to choose a strategy (Erev and Roth 1998). The modified update function MUF is based on Nicolaisen et al. (2001) and depends on the chosen strategy $s^{\prime}$ in $t$ :

$$
\operatorname{MUF}\left(i, s, s^{\prime}, t\right)=\left\{\begin{array}{cc}
\Pi_{i}(t)(1-\exp ), & s=s^{\prime} \\
q_{i s}(t) \frac{\exp }{|S|-1}, & s \neq s^{\prime}
\end{array} .\right.
$$


The experiment parameter exp determines the weight of impact of, firstly, the profit on the future propensity, and secondly, the reduction of the propensities of the strategies that were not currently chosen (Erev and Roth 1998). The probabilities to choose a certain strategy are updated according to:

$$
p_{i s}(t+1)=\frac{q_{i s}(t+1)}{\sum_{s=s_{1}}^{|S|} q_{i s}(t+1)}
$$

The probability to choose a strategy $s$ in the first time slot to submit the price for an agent's bid to the market in $t+1$ is set to $p_{i s}\left(t_{0}\right)=\frac{1}{|S|}$. Accordingly, agents randomly choose their strategy in $t_{0}$. After the market clearing house has matched the bids and offers from the first matchable time slot, agents now know their payed and received prices in that time slot and can calculate their profit, update their propensities and probabilities, accordingly. Like this, the Erev-Roth learning approach solely focuses on profit as the agents' objective utility function. Preferences of energy source or locality (Mengelkamp and Weinhardt 2018) are not taken into account. The Erev-Roth algorithm runs individually for each agent and gets updated in every time slot.

\section{Demand shifting for DR}

In addition to trading on the LEM, agents also have the possibility to shift (part of) their demand to future time slots. The main aim of our DR strategy is to reduce the aggregated peak demand of the residential agents on the LEM level. Originally, we implemented the DR algorithm of Albadi and El-Saadany (2008). This work is based on dynamic grid pricing according to the forecasted price elasticity of demand. The price elasticity of demand is driven by economic benefits, and as a result consumers shift their demand to periods with lower grid prices.

However, this strategy has a large drawback in our setup with a constant reference tariff, as dynamic grid pricing is not applied in our LEM simulation. We observed sudden spikes in the aggregated demand profile of the LEM with DR. To mitigate this challenge we develop a modified DR strategy based on the existing strategy of Albadi and El-Saadany (2008). Figure 2 shows the flow chart of the modified DR algorithm. Under this modified strategy, the individual households agents $I=\{1,2, \ldots, N\}$ forecast their demand profile (perfect forecast) for $15 \mathrm{~min}$ intervals for the next $24 \mathrm{~h}$ as $D_{i}=\left\{d_{i, t_{0}}, d_{i, t_{1}}, \ldots, d_{i, t_{95}}\right\}$. With the demand profile for the next $24 \mathrm{~h}$, an agent $i \in I$ forecasts the maximum peak of its demand in that $24 \mathrm{~h}$ interval through the maximization function $D_{i}\left(d_{i, \max }\right)$ as

$$
D_{i}\left(d_{i, \max }\right)=\max \left(D_{i}\left(d_{i, t}\right)\right), t=t_{0}, t_{1}, \ldots, t_{95} .
$$

Then, a shaded DR parameter $S D R$ is calculated depending on the realistic forecast of how much of the demand can be shifted during a time interval. The SDR determines the limit above which demand can be shifted to another time of the day. This means that all the peaks in the interval which are higher than a certain percentage of the maximum peak determined by the $S D R$, are shifted to the troughs of the demand profile. With a $S D R=[0,1]$, DR applies to all the demand points $D_{i}\left(d_{i, t}\right)$ which satisfy the following condition

$$
D_{i}\left(d_{i, t}\right)>D_{i}\left(d_{i, \max }\right) * S D R, t=t_{0}, t_{1}, \ldots, t_{95} .
$$

The demand intervals which satisfy the above condition are denoted by $D_{i}^{\text {shift }}\left(d_{i, t(j)}\right), j=1,2, . . n$, where $n$ is the number of peaks above the SDR threshold, and 


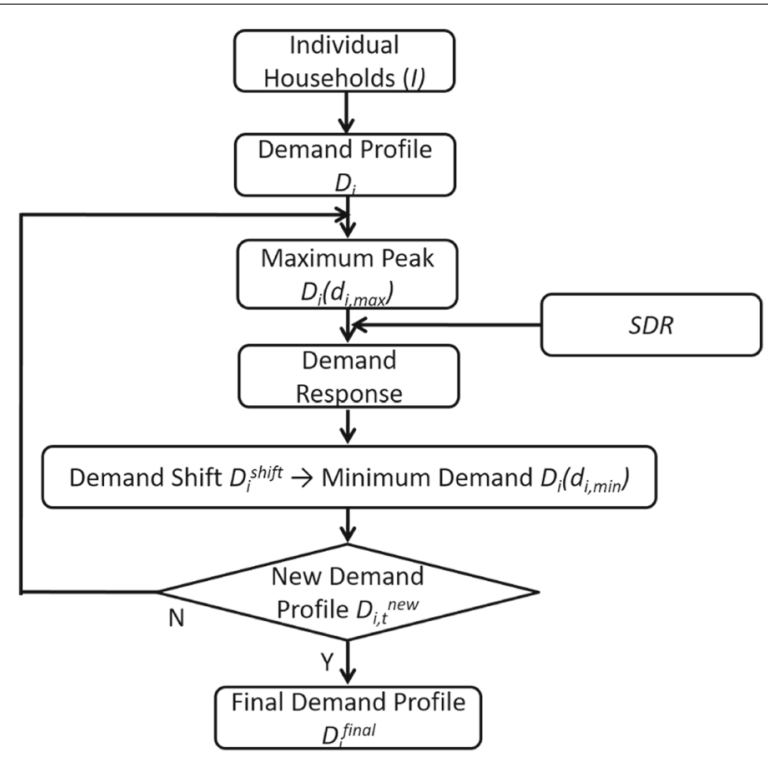

Fig. 2 Modified DR strategy. Figure 2 shows the modified DR strategy implemented in our simulation. Each household $i \in /$ forecasts his demand $D_{i}$ in 15 min time steps for the next $24 \mathrm{~h}$. Then, the maximum peak demand $D_{i}\left(d_{i, \max }\right)$ is determined from the forecasted demand $D_{i}$. The SDR determines the shiftable demand $D_{i}^{\text {shift. }}$. It is determined as all demand in time slots $t(j)$ that is above the $S D R$. The first timeslot $t\left(j_{1}\right)$, in which a shiftable demand is determined, is then shifted towards the current minimum of the forecasted demand $D_{i}\left(d_{i, \min }\right)$. Thus, a new demand profile $D_{i, t}^{\text {new }}$ is created. If the new demand profile is final, i.e. iterated for 96 times, $D_{i, t}^{\text {new }}=D_{i}^{\text {final }}$. If not, another iteration is started

the values of $D_{i}^{\text {shift }}\left(d_{i, t(j)}\right)$ are given by the following equation

$$
D_{i}^{\text {shift }}\left(d_{i, t(j)}\right)=D_{i}\left(d_{i, t(j)}\right)-D_{i}\left(d_{i, \text { max }}\right) * S D R, t(j)=t\left(j_{1}\right), t\left(j_{2}\right), \ldots, t\left(j_{n}\right) .
$$

The index $j$, hereby, determines the demand points of agent $i$ that are above SDR. Once the values of $D_{i}^{\text {shift }}\left(d_{i, t(j)}\right)$ are determined for all applicable agents, the amount of energy that is to be shifted from the individual time intervals is determined.

In the next step, we determine the minimum demand from the forecasted demand profile $D_{i}\left(d_{i, t}\right)$. The minimum demand point denoted as $D_{i}\left(d_{i, \text { min }}\right)$ per agent gives the minimum demand of a particular household for the next $24 \mathrm{~h}$. Another parameter which is important for demand shifting is the time interval $t_{i, \min }$ when $D_{i}\left(d_{i, \text { min }}\right)$ occurs. The minimum demand point is determined by the following equation

$$
D_{i}\left(d_{i, \min }\right)=\min \left(D_{i, t}\right), t=t_{0}, t_{1}, \ldots, t_{95} .
$$

Once $D_{i}\left(d_{i, \text { min }}\right)$ is established, the first demand shift takes place where the $D_{i}^{\text {shift }}\left(d_{i, j_{1}}\right)$ is moved to the time interval which is determined by $t_{i, \text { min }}$. Once these steps took place, the new demand profile with the demand shift is determined as $D_{i, t}^{\text {new }}\left(d_{t_{0}}, d_{t_{1}}, \ldots, d_{t_{95}}\right)$. Once this is achieved, the DR strategy loops back to determining the new maximum peak demand according to Fig. 2. These iterations are happening each time slot for every agent for a maximum amount of 96 times. The goal is to create a minimum peak final demand curve $D_{i}^{\text {final }}$ for every agent. Once the $D_{i}^{\text {final }}$ is established, the modified final demand profile is sent back to the individual households as an input for participation in the trading process of the LEM. This method focuses on load shifting without any energy losses or gains during the shifting process. 


\section{Key performance indicators}

We assess the results of the simulations of our LEM scenario based on selected key performance indicators (KPIs). These KPIs allow to compare our test set of scenarios, which differ regarding underlying regulation and degree of interactions among the market participants. The KPIs support the extraction of information about the economic and physical impact of LEM trading and DR, both for the agents and the physical electricity grid in which the LEM is embedded. We consider the following three KPIs reported on an aggregated annual market level:

1. Degree of Local Sufficiency (DLS)

2. Market Closing Price (MCP)

3. Residual Peak Demand (RPD)

The DLS is an indicator for the degree to which a LEM can be self-sufficient (or autark) compared to the aggregated demand of all agents. Therefore, DLS is defined as the sum of energy instantaneously self-consumed $s c_{i, t}$ by the sum of all agents $i \in I$ in the LEM and the energy successfully traded $e t_{i, t}$ on the LEM, divided by the aggregated original demand $\sum_{i=1, t=t_{0}}^{N, T} d_{i, t}^{\text {original }}$ in the LEM. The constant $T$ states the maximum amount of time slots considered over one year, i.e. 35040 time slots. The original demand does not include any DR.

$$
D L S=\sum_{i=1}^{N} \sum_{t=t_{0}}^{T} \frac{s c_{i, t}+e t_{i, t}}{d_{i, t}^{\text {original }}}
$$

The MCP is an indicator for the economic gain or loss of trading on the LEM as compared to electricity supply from the public grid. The MCP is defined as the average weighted uniform market closing price over all LEM electricity trades per year.

$$
M C P=\frac{\sum_{i=1, t=t_{0}}^{N, T} M C P_{t} e t_{i, t}}{\sum_{i=1, t=t_{0}}^{N, T} e t_{i, t}} .
$$

The RPD relates to the maximum residual peak demand in a given time interval aggregated over all agents after self-consumption, trading on the LEM and DR. It is thus an indicator for the degree to which the LEM still depends on an overarching grid infrastructure although local RES, a LEM and DR are available. The necessary demand function for calculating the RPD is the forecasted demand $D_{i, t}$. It equals $D_{i, t}^{\text {original }}$ for scenarios without DR and $D_{i, t}^{\text {final }}$ for scenarios with DR. As we assume a perfect short term forecast, ex post deviations do not apply.

$$
R P D=\max \left\{\begin{array}{c}
\sum_{i=1}^{N} D_{i, t_{0}}-\left(s c_{i, t_{0}}+e t_{i, t_{0}}\right) \\
\sum_{i=1}^{N} D_{i, t_{1}}-\left(s c_{i, t_{1}}+e t_{i, t_{1}}\right) \\
\cdots \\
\sum_{i=1}^{N} D_{i, T}-\left(s c_{i, T}+e t_{i, T}\right)
\end{array} .\right.
$$

\section{Set of scenarios}

We distinguish our set of scenarios, firstly, with regard to the regulatory situation in which a LEM could be operated in the German context and secondly, with regard to the degree 
of interactions between the different agents. We identify two existing and one idealized regulatory framework for a LEM:

1. Virtual community of agents interacting on the public grid

2. Physical community of agents interacting in a microgrid ${ }^{3}$

3. Physical community of agents interacting under a favorable regulatory framework

The regulatory framework defines the cost at which electricity can be offered by prosumers for trading on the LEM. It is highest when using the public grid, as the entirety of regulated German tariff components has to be paid in addition to an opportunity cost from a lost feed-in tariff. Prosumers in Germany are currently guaranteed a specific feedin tariff $c^{F}$ for RES feed-in into the public grid. Thus, this opportunity cost with the addition of taxes, fees and levies stipulates the lower cost bound for selling RES generation on the LEM. In a physical microgrid certain regulated tariff components do not apply. This decreases the possible offer price and opens up a trading window as compared to the national reference tariff of the public grid. With the third case of a favorable regulatory framework for LEM trading, we study the impact of omitting the renewable surcharge in a LEM. A detailed overview of the impact of the regulatory framework on possible bid prices is given by Table 1 . In a public grid, trading on the LEM would only be possible above the reference tariff. As we only consider LEM trading between the feed-in tariff plus regulated tariff components and the reference tariff, trading will not be economic and is thus not applied in our simulation. However, both the microgrid and favorable regulation scenario offer a trading window between the considered lower and upper LEM price bounds.

We combine each of the regulatory scenarios with four degrees of interaction among the agents shown in Table 2. In the base case we assume that prosumers maximize their selfconsumption and feed-in excess generation while no LEM trading or DR takes place. This is the reference case, which represents the current situation for prosumers in Germany. In the trading case, we assume that in addition to self-consumption, prosumers bid any residual generation on the LEM. This allows consumers to procure electricity on the LEM. For the trading and DR case, we assume that, in addition to the trading scenario, all agents apply a DR strategy as defined in section "Demand shifting for DR". Thus, agents may trade on the LEM and may shift their demand to future time slots. For reference,

Table 1 Description of regulatory scenarios (prices in $c € / \mathrm{kWh}$ )

\begin{tabular}{llll}
\hline Regulatory scenario & Public grid & Microgrid & Favourable regulation \\
\hline Reference tariff & 29.86 & 29.86 & 29.86 \\
Grid fees & 6.99 & - & - \\
Metering \& concession fees & 1.94 & 1.94 & 1.94 \\
Renewable surcharge & 6.88 & 6.88 & - \\
Grid related fees & 0.81 & - & - \\
Electricity tax & 2.05 & - & - \\
Opportunity cost PV & 12.20 & 12.20 & 12.20 \\
Opportunity cost CHP & 11.42 & 11.42 & 11.42 \\
Subtotal (PV/CHP) & $30.87 / 30.09$ & $21.02 / 20.24$ & $14.14 / 13.36$ \\
Value-added Tax (19\%) & $5.87 / 5.72$ & $3.99 / 3.85$ & $2.69 / 2.54$ \\
Lowest bid price (PV/CHP) & $36.74 / 35.81$ & $25.01 / 24.09$ & $16.83 / 15.90$ \\
\hline
\end{tabular}


Table 2 Degree of interaction among the agents

\begin{tabular}{lllll}
\hline & Base & Trading & Trading + DR & Upper Bound \\
\hline Self-Consumption & Yes & Yes & Yes & Yes \\
Trading & None & Yes & Yes & Enforced \\
DR & None & None & Yes & Yes \\
\hline
\end{tabular}

we also provide an upper bound scenario. Here, we assume that consumers always bid an offer price equal to the grid reference tariff, instead of adapting their bids with the pricing strategy from section "Pricing strategy". Thus, the maximum amount of trading is realized.

\section{Simulation results}

\section{Setup of simulation}

The simulation is set up according to the model methodology explained in section "Model methodology" and Fig. 1. In AnyLogic the simulation starts up with a main class that is initiating all simulated agents as prosumers. A consumer is initiated as a prosumer with zero generation. Further, the main class inserts the external generation and demand data into the model in every simulation time slot (i.e. simulated $15 \mathrm{~min}$ ). The generation and demand data is added to every simulated agent. Each consumer/prosumer agent has 2 main functions, that implement strategies for their decision making. Firstly, the pricing strategy (described in section "Pricing strategy"), and secondly, the DR shifting strategy (described in section "Demand shifting for DR"). If the pricing strategy is enabled, agents learn from their past trades and adapt their bids (offers) accordingly. If it is disabled, no bids and offers are generated. Enabling the DR shifting strategy results in DR being conducted for the agents. DR shifting is executed before the pricing strategy, and the modified demand $D_{i}^{\text {final }}$ of the DR is used as input for creating the bids and offers.

If the pricing strategy is enabled, the bids and offers are send to the clearing house, where the market mechanism is conducted. The resulting successful orders are being send back to the respective agents in terms of matched amounts of energy and market closing price. All unsuccessful bids and offers are being transferred to the grid as back-up option before returned to the agents.

The different regulatory scenarios are implemented by adapting the lower and upper price limit of the agents' pricing strategy according to the regulatory scenario (cf. Table 1). The interaction scenarios are realized through enabling (disabling) the pricing and DR shifting functions for the agents according to the targeted interaction scenario. For the base scenario, neither the pricing function nor the DR shifting function are enabled. The trading scenario activates the pricing function. The trading and DR scenario activate both, the pricing and the DR shifting function. For the upper bound scenario, DR shifting is activated, and pricing is enforced. The enforcement of pricing hereby means, that agent bids (offers) are enforced to be at the maximum (minimum) price limit for each agent. This results in the highest amount of trading that is feasible. We evaluate completed simulation runs over one year with the respective KPIs introduced in section "Key performance indicators". The KPIs are calculated directly in AnyLogic by an implemented evaluation function. 


\section{Data origin}

The PV data is based on a measured time series from $5 \mathrm{kWp}$ PV panels in Southern Germany in 15 min time slots for the year 2013. It was used to generate the data for electricity production for the population of prosumer agents with a $20 \%$ uniform distributed randomization function. The CHP data is based on multi-year measured time series collected from nine residential CHP installations of 0.7-1 kWp installed electrical power, thereof one located in Southern Germany, one in Alsace (France) and seven in Fontainebleau (France). The PV and CHP data was collected in the frame of research and development projects and is not publicly available.

The household consumption data is based upon standard demand profiles (Stadtwerke Unna 2002) and adapted with the same randomized error function that was used for the generation data to fit 1-5 person households.

\section{Test runs}

We conduct 10 test runs in 15 min time slots with 100 residential agents over the duration of 1 year for every scenario. For each test instance, the generation and demand data is newly instantiated according to the following section "Data origin". Furthermore, the pricing strategy from section "Pricing strategy" and the DR strategy from section "Demand shifting for DR" are newly instantiated for each each agent in each test run. The pricing strategy is implemented with a scaling, recency and experimental parameter of $s c a=1.0, r e c=0.02$ and $\exp =0.99$ as taken from Nicolaisen et al. (2001). The $S D R$ is set to 0.7. The test runs were conducted on a standard laptop computer with Intel(R) Core(TM) i5-6200U Processor CPU @ 2.30GHz, 2400 Mhz, 2 Core(s), 4 Logical Processor(s) along with 8.00GB RAM. The simulation was executed in the AnyLogic 8.1.0 personal learning edition. One simulation run over 1 year in 35040 time slots took on average $20 \mathrm{~min}$.

\section{Evaluation}

While we evaluate the simulation test runs, we focus on the LEM as a whole and analyze the DLS, MCP and RPD averaged yearly over all conducted test runs per scenario (see section "Test runs"). The comparison of the DLS between the four interaction and three regulatory scenarios is depicted in Fig. 3. In the base case scenario, no trading is conducted, thus the DLS stays at $22 \%$, which is the amount of direct self-consumption by the prosumers. This direct self-consumption is constant for all regulatory scenarios. Direct self-consumption includes the self-consumption by PV and CHP prosumers. In the trading scenario on a public grid, no trading happens due to the missing trading window, as explained in section "Set of scenarios". The microgrid scenario allows for a small trading window, increasing the DLS by less than $0.5 \%$. The favorable regulation scenario allows for a significant amount of trading on the LEM, due to the regulatory freedom allowing a significant price gap between the minimum offer prices and the reference tariff. This increases the DLS by $10 \%$.

As DR is introduced in addition to trading on the LEM, both, the public grid and microgrid scenario increase the DLS by $7 \%$ due to demand shifting towards times in which self-consumption can be done. The DLS is increased by additional $3 \%$ in the favorable regulation scenario, if DR is added to the trading scenario. This is due to the favorable regulation already providing a high amount of DLS in the trading scenario. Thus, the increase is less significant in absolute terms than for the public grid and microgrid scenario. 


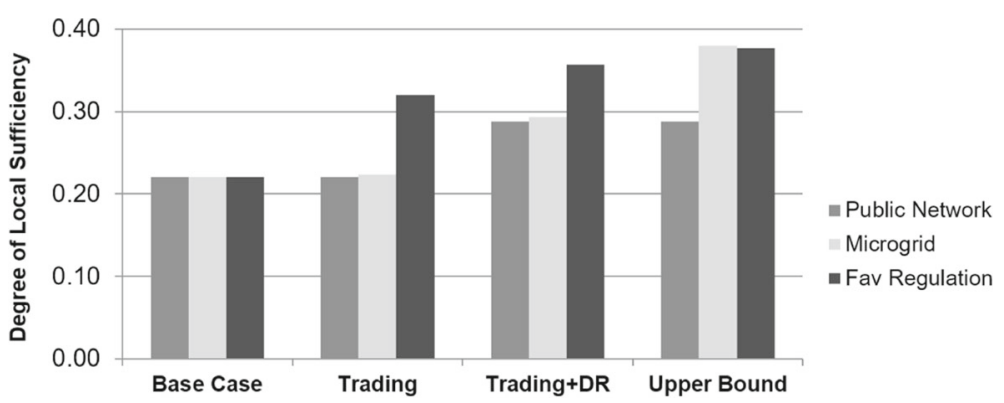

Fig. 3 Degree of local sufficiency. Figure 3 shows the simulated DLS dependent on the 4 interaction scenarios as well as the 3 regulatory scenarios that we consider. In the base case interaction scenario, trading is not allowed and only direct self-consumption drives the DLS to $22 \%$. In the trading scenario, the public grid allows no trading due to a non-existent price spread. The microgrid scenario increases the DLS by $0.5 \%$ due to a minimal price spread. However, the favorable regulation scenario introduces a large price spread and allows an increase of the DLS by $10 \%$. Including DR allows increasing the DLS significantly in all interaction scenarios. Here, the maximum DLS is reached in the favorable regulation scenario, as both trading with a large price spread and DR are possible. The upper bound scenario shows the maximum theoretically feasible DLS with enforced maximum trading. The theoretically feasible DLS lies at 38\%. This is almost reached by the trading and DR scenario in case of the favorable regulation scenario

The upper bound scenario shows the maximum feasible DLS for all regulatory cases. Maximal trading in a public grid results in a DLS of $29 \%$, which is already reached by the scenario combining trading and DR. The maximal DLS for the microgrid and favorable regulation scenario, however, lies at $38 \%$ and is not yet reached by the other interaction scenarios, as a part of the LEM trading is not yet conducted, due to agents bidding below the maximum price limit, and thus reducing LEM trading. The analysis shows that under current German regulation, the participation in a LEM with competitive price determination and DR may increase the DLS by about $7 \%$.

Figure 4 shows the MCP for all scenario combinations. In the base case, no trading on the LEM exists, and thus, no MCP can be calculated, just as in the public grid case of all interaction scenarios. The agents in both, the trading and the trading with DR scenarios, do not always bid their maximum price limit, but rather bid intelligently. Thus less trading happens and, consequently, the DLS is lower than in the upper bound scenario, as shown in Fig. 3. The MCP is slightly decreasing from the trading to the trading and DR scenario for both the microgrid and the favorable regulation scenario. This is due to demand being shifted towards self-consumption. Thus, less demand is left for trading and the LEM follows the rules of the market to lower the prices in case demand is decreasing with a constant supply. In the upper bound scenario, the MCP in the microgrid and favorable regulation scenario are higher than in all other scenarios. This is due to fixed offer prices by the agents. As prices are fixed at the maximum valuation for buyers, a high MCP is reached in all time slots.

In all interaction scenarios, the MCP is always higher in the microgrid scenario than in the favorable regulation scenario. This is due to the favorable regulation scenario offering a larger price margin for trading. Lower MCPs are reached and the average MCP decreases. This gap between the MCP in the microgrid and favorable regulation scenario is smallest in the upper bound, where high buy prices are enforced, which is to the benefit of producers. 


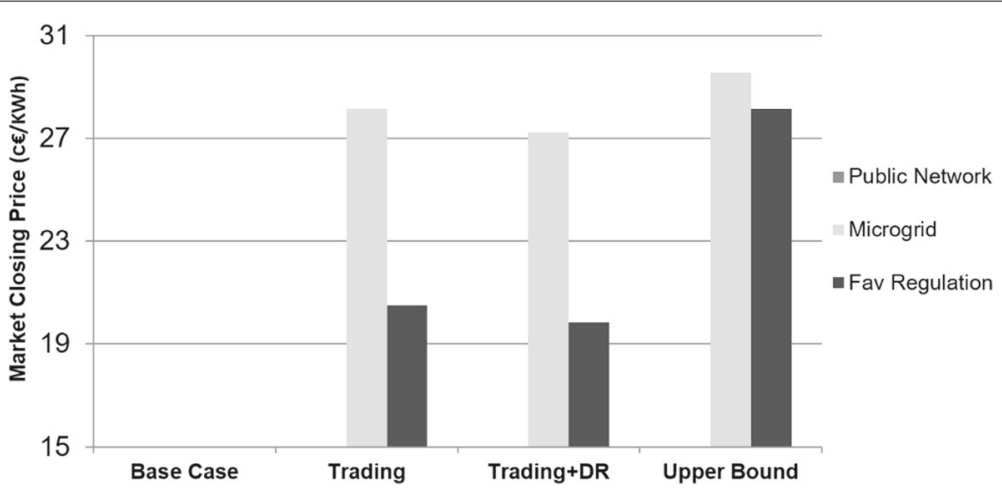

Fig. 4 Average market closing price. Figure 4 shows the MCP for the 4 interaction and 3 regulatory scenarios that we consider. In case of the base case and the public grid, no trading is feasible, thus, no MCP can be calculated. When trading takes place, the MCP is decreasing from the microgrid to the favorable regulation scenario for all interaction scenarios. This is due to the favorable regulation scenario offering a larger price spread for trading at the same supply and demand scenario. Thus, prices are decreasing on the LEM. Including DR to the trading scenario results in the same behavior, i.e. a decrease in the MCP. This time, the decrease results from demand being shifted from peaks, and thus creating less scarcity scenarios that would result in higher MCPs. The upper bound scenario results in the highest MCPs due to enforced high bidding. All in all, the MCP may be reduced by up to $32 \%$ compared to the reference tariff in the simulation

The annual RPD in the simulation is depicted in Fig. 5. We simulate the demand in kW per 15 min time slot. Assuming that the power is equally distributed per time slot, we report the peak demand per year in Fig. 5. As shown there, trading on the LEM does not influence the residual peak demand. However, DR aims at reducing peaks, and thus, naturally, has a large influence on the RPD. The RPD in the public grid scenario is always slightly higher than in the microgrid and favorable generation scenarios for the DR interaction scenarios. This is due to no trading happening in the public grid scenario. Thus, in the public grid scenarios, the entire amount of electricity is procured from the grid. This increases the RPD. We show that solely trading on the LEM has no influence on the RPD in any of the regulatory scenarios. Thus, there is no physical influence on the grid

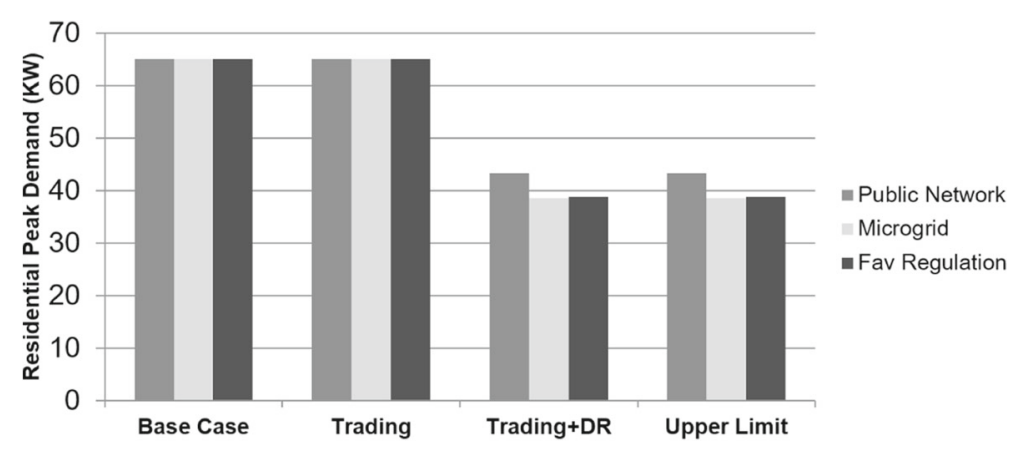

Fig. 5 Annual residual peak demand. Figure 5 shows the RPD for the 4 interaction and 3 regulatory scenarios that we consider. Assuming that power consumption is equally distributed per time slot, the maximum residual peak demand depicts the peak power demand from the LEM towards the superimposed grid. In the base case and the trading case the RPD is constant, as it is not influenced by solely virtual trading. However, the objective of DR is decreasing the power peaks. This works best in the microgrid and favorable regulation scenario. The public grid scenario still results in higher RPDs than the microgrid and favorable regulation scenario due to all electricity being procured from the superimposed grid. When trading offers a significant price spread, however, the RPD can be further decreased (see microgrid and favorable regulation scenario). The combination of LEM and DR may reduce the RPD by up to $40 \%$ 
operation. Combining LEM with DR, however, offers the chance to reduce the RPD for all regulatory scenarios. Thus, the combination of LEM and DR fulfills this objective and may reduce the RPD by up to $40 \%$.

\section{Discussion}

The conducted simulation runs show, how the DLS can be increased by adding DR in all regulatory scenarios, and by adding LEM trading in the microgrid and favorable regulation scenario. The DLS can be increased to up to $36 \%$. This is only a $9.5 \%$ gap to the upper bound. Likewise, the MCP for trading can be reduced by up to $32 \%$ while the RPD can be reduced by up to $40 \%$ if DR is incorporated in the simulation. Our DR strategy was calculated with a $S D R$ of $70 \%$. This means, that $30 \%$ of the residential load was flexible and could be shifted to future time slots within the DR strategy. However, a LEM is a solely virtual market without any operational influences on the physical grid. Thus, only the combination of the two allows end customers to positively influence the RPD.

Because of the increasing decentralization of electricity generation capacity, the introduction of LEM should offer the opportunity for grid-supportive operation. Current levels of control and redispatch measures on transmission and distribution levels as well as the associated costs show that the grid-supportive operation of decentralized energy systems are becoming a key factor in the German energy system. LEMs may support the grid integration of decentralized generation technologies, if combined with DR or other incentives striving for grid-supportive operational patterns.

It is important to note that there are several limitations in the simulated data with regards to the general applicability in real world case studies. First of all, the household demand is based on standard consumption profile. Although these were randomized accordingly (see section "Data origin"), measured data (with higher resolutions than $15 \mathrm{~min}$ ) should be taken and tested with the simulation model to validate the results. With high resolution profiles, the real (and not averaged) peak could be better considered. As real profiles also offer more variability than standard profiles, the potential for demand shifting would be higher. Thus, we can derive that our simulation represents a lower bound of DR potential.

Furthermore, the idea of a LEM with the established reference tariff as back-up option is a representation of pilot and test cases of LEMs. The sustainable implementation of LEMs as an addition or alternative to the existing German energy market will require a revision of the socialization of grid fees, and an establishment of inter-LEM trading and energy transmission. Additionally, the role of energy storage in LEMs and DR needs to be extensively integrated in further research. Energy storage may offer significant benefits for the level of self-sufficiency and grid-supportive operation.

\section{Conclusion \& outlook}

This work presents a comprehensive simulation study of a LEM in combination with DR in the German energy market context. We present LEMs as a way to decentralize the wholesale energy market in line with the ongoing distribution and decentralization of renewable generation. We developed an agent-based simulation in which for both, the pricing strategy and the demand shifting strategy, appropriate models from literature were adapted and integrated to our LEM concept. With this model, we compare the level of self-sufficiency, electricity prices and residual annual peak demand of two 
interaction scenarios, i.e. LEM trading and LEM trading with DR. To set a frame of reference, we also provide a base case and an upper bound case for our LEM scenario. Each of these LEM scenarios is considered to operate according to regulations for the public grid, a regulatory niche for a microgrid approach, and a proposed favorable regulation approach. The results show that self-sufficiency may be increased by up to $16 \%$, the electricity price can be reduced by up to $32 \%$ and the residual aggregated peak demand of our set of agents can be reduced by up to $40 \%$, depending on the regulatory scenario. In consequence, the combination of LEM and DR may allow for more self-sufficient energy communities on a distribution level. Such communities may procure electricity locally at advantageous prices and, on an aggregated level, consume and inject RES generation in a grid-supportive way from and to the public grid. Hence, congestion management requirements may be reduced at the distribution and transmission grid levels in the long term.

Concerning further research points, besides the German context prevailing in this study, a transfer to other countries and their corresponding regulations could allow to verify a more general validity of our conclusions. Furthermore, our pricing strategy is based on rational economic criteria whereas in the real world agent preferences are also influenced by non purely economic aspects (e.g. preference for locally produced electricity, preference for renewable energies). An extension to include non-economic agent preferences should thus be further considered.

\section{Endnotes}

${ }^{1}$ The current version of the EnWG can be found here: http://www.gesetze-im-internet. de/enwg_2005/index.html (Accessed 23 July 2018).

${ }^{2}$ The EEX is a central market place for energy and related commodities for over 30 countries: https://www.eex.com/de/about/eex/eex-ag (Accessed 19 Apr 2018).

${ }^{3} \mathrm{~A}$ microgrid is derived following the areal grid "Kundenanlage" in $\$ 324 \mathrm{a}-\mathrm{b}$ Energiewirtschaftsgesetz (EnWG).

Abbreviations

ABM: Agent-based modelling; CHP: Combined heat-and-power; DLS: Degree of local sufficiency; DR: Demand response; DSM: Demand side management; EE: Energy efficiency; KPI: Key performance indicators; LEM: Local energy market; MCP: Market closing price; PV: Photovoltaic; RES: Renewable energy sources; RPD: Residual peak demand; SR: Spinning reserve; TOU: Time-of-use tariff

Acknowledgements

The authors' gratefully acknowledge the support of Prof. Dr. Ute Karl at EIFER and Christoph Reeber.

Funding

The paper was partly funded by the Helmholtz project "Storage and Cross-linked Infrastructure (SCI)" at KIT and by EDF $R \& D$.

Availability of data and materials

The datasets used and/or analyzed during the current study are available from the corresponding author on reasonable request. The PV generation data is not publicly available as it is self-measured over the time of several projects. CHP data used in this study originates from field tests in cooperation with EDF R\&D and is therefore not publicly available. The household demand data is based upon the standardized data sets by Stadtwerke Unna (2002).

Authors' contributions

All authors made substantial contributions to conception, design, acquisition, analysis, the interpretation of the analyzed data and writing the manuscript. All authors read and approved the final manuscript.

Competing interests

The authors declare that they have no competing interests.

\section{Publisher's Note}

Springer Nature remains neutral with regard to jurisdictional claims in published maps and institutional affiliations. 


\section{Author details}

${ }^{1}$ Karlsruhe Institute of Technology, Fritz-Erler-Str. 23, 76131 Karlsruhe, Germany. ${ }^{2}$ European Institute for Energy Research, Emmy-Noether-Str. 11, 76131 Karlsruhe, Germany.

\section{Received: 3 July 2018 Accepted: 2 August 2018}

Published online: 22 August 2018

\section{References}

Albadi MH, El-Saadany EF (2008) A summary of demand response in electricity markets. Electr Power Syst Res 78(11):1989-1996

Ampatzis M, Nguyen PH, Kling W (2014) Local electricity market design for the coordination of distributed energy resources at district level. In: Innovative Smart Grid Technologies Conference Europe (ISGT-Europe), 2014 IEEE PES. IEEE. pp 1-6

Babic J, Podobnik V (2016) A review of agent-based modelling of electricity markets in future energy eco-systems. In: Computer and Energy Science (SpliTech), International Multidisciplinary Conference on. IEEE. pp 1-9

Bauwens T (2016) Explaining the diversity of motivations behind community renewable energy. Energy Policy 93:278-290

Buchmann E, Kessler S, Jochem P, Böhm K (2013) The costs of privacy in local energy markets. In: Business Informatics (CBI), 2013 IEEE 15th Conference on. IEEE. pp 198-207

Cutter E, Woo CK, Kahrl F, Taylor A (2012) Maximizing the value of responsive load. Electr J 25(7):6-16

de Durana JMG, Barambones O, Kremers E, Varga L (2014) Agent based modeling of energy networks. Energy Conversion and Management 82:308-319

Erev I, Roth AE (1998) Predicting how people play games: Reinforcement learning in experimental games with unique, mixed strategy equilibria. Am Econ Rev:848-881

Gordijn J, Akkermans H (2007) Business models for distributed generation in a liberalized market environment. Electr Power Syst Res 77(9):1178-1188

Gottwalt S, Gärttner J, Schmeck H, Weinhardt C (2017) Modeling and valuation of residential demand flexibility for renewable energy integration. IEEE Trans Smart Grid 8(6):2565-2574

Green J, Newman P (2017) Citizen utilities: The emerging power paradigm. Energy Policy 105:283-293

Hochschule für Technik Stuttgart (2016) Sim4Blocks. http://www.sim4blocks.eu/. Accessed 26 June 2018

Hvelplund F (2006) Renewable energy and the need for local energy markets. Energy 31(13):2293-2302

Ilic D, Da Silva PG, Karnouskos S, Griesemer M (2012) An energy market for trading electricity in smart grid neighbourhoods. In: Digital Ecosystems Technologies (DEST), 2012 6th IEEE International Conference on. IEEE. pp 1-6

Intergovernmental Panel on Climate Change (IPCC) (2011) Special report on renewable energy sources and climate change mitigation. Cambridge University Press, New York

Jia NX, Yokoyama R (2003) Profit allocation of independent power producers based on cooperative game theory. Int Electr Power Energy Syst 25(8):633-641

Kamrat W (2001) Modeling the structure of local energy markets. IEEE Comput Appl Power 14(2):30-35

Koirala BP, Koliou E, Friege J, Hakvoort RA, Herder PM (2016) Energetic communities for community energy: A review of key issues and trends shaping integrated community energy systems. Renew Sust Energ Rev 56:722-744

Kremers EA (2013) Modelling and simulation of electrical energy systems through a complex systems approach using agent-based models. Universidad del Pais Vasco, Spain, Karlsruhe

Marzband M, Sumper A, Domínguez-García JL, Gumara-Ferret R (2013) Experimental validation of a real time energy management system for microgrids in islanded mode using a local day-ahead electricity market and MINLP. Energy Convers Manag 76:314-322

Marzband M, Ghadimi M, Sumper A, Domínguez-García JL (2014) Experimental validation of a real-time energy management system using multi-period gravitational search algorithm for microgrids in islanded mode. Appl Energy 128:164-174

Marzband M, Javadi M, Domínguez-García JL, Moghaddam MM (2016) Non-cooperative game theory based energy management systems for energy district in the retail market considering DER uncertainties. IET Generation. Transm Distrib 10(12):2999-3009

Mengelkamp E, Gärttner J, Rock K, Kessler S, Orsini L, Weinhardt C (2018a) Designing microgrid energy markets: A case study: The Brooklyn Microgrid. Appl Energy 210:870-880

Mengelkamp E, Gärttner J, Weinhardt C (2018) Decentralizing energy systems through local energy markets: The LAMP-Project. MKWI

Mengelkamp E, Staudt P, Garttner J, Weinhardt C (2017) Trading on local energy markets: A comparison of market designs and bidding strategies. In: European Energy Market (EEM), 2017 14th International Conference on the. IEEE. pp 1-6

Mengelkamp E, Weinhardt C (2018) Clustering household preferences in local electricity markets. In: Proceedings of the Ninth International Conference on Future Energy Systems. ACM, NY. pp 538-543

MWS Projektentwicklungsgesellschaft mbH (2018) FRANKLIN Mannheim Project. http://franklin-mannheim.de/. Accessed 15 May 2018

Nicolaisen J, Petrov V, Tesfatsion L (2001) Market power and efficiency in a computational electricity market with discriminatory double-auction pricing. IEEE Trans Evol Comput 5(5):504-523

Olivella-Rosell P, Lloret-Gallego P, Munné-Collado Í, Villafafila-Robles R, Sumper A, Ottessen SØ, Rajasekharan J, Bremdal BA (2018) Local flexibility market design for aggregators providing multiple flexibility services at distribution network level. Energies 11(4):822

Palensky P, Dietrich D (2011) Demand side management: Demand response, intelligent energy systems, and smart loads. IEEE Trans Ind Inform 7(3):381-388

Pinson P, Madsen H (2014) Benefits and challenges of electrical demand response: A critical review. Renew Sust Energ Rev 39:686-699

Prüggler N (2013) Economic potential of demand response at household level_Are Central-European market conditions sufficient? Energy Policy 60:487-498 
Rosen C, Madlener R (2012) An auction mechanism for local energy markets: Results from theory and simulation. In: Complexity in Engineering (COMPENG), 2012. IEEE. pp 1-4

Rosen, C, Madlener R (2013) An auction design for local reserve energy markets. Decis Support Syst 56:168-179

Sæle H, Grande OS (2011) Demand response from household customers: Experiences from a pilot study in Norway. IEEE Trans Smart Grid 2(1):102-109

Sensfuß F, Ragwitz M, Genoese M, Möst D (2007) Agent-based simulation of electricity markets: a literature review (No S5/2007). Report, Fraunhofer ISI

Shariatzadeh F, Mandal P, Srivastava AK (2015) Demand response for sustainable energy systems: A review, application and implementation strategy. Renew Sust Energ Rev 45:343-350

Siano P (2014) Demand response and smart grids_-A survey. Renew Sust Energ Rev 30:461-478

Singh H (1999) Introduction to game theory and its application in electric power markets. IEEE Comput Appl Power 12(4):18-20

Stadtwerke Unna (2002) VDEW-BDEW-Lastprofile. http://tinyurl.com/j4qa7qb. Accessed 20 June 2018

Tveten $\AA$ G, Bolkesjø TF, Martinsen T, Hvarnes H (2013) Solar feed-in tariffs and the merit order effect: A study of the German electricity market. Energy Policy 61:761-770

Vytelingum P, Ramchurn SD, Voice TD, Rogers A, Jennings NR (2010) Trading agents for the smart electricity grid. In: Proceedings of the 9th International Conference on Autonomous Agents and Multiagent Systems: volume 1-Volume 1. pp 897-904. International Foundation for Autonomous Agents and Multiagent Systems

Yousefi S, Moghaddam MP, Majd VJ (2011) Optimal real time pricing in an agent-based retail market using a comprehensive demand response model. Energy 36(9):5716-5727

Zhou Z, Zhao F, Wang J (2011) Agent-based electricity market simulation with demand response from commercial buildings. IEEE Trans Smart Grid 2(4):580-588

\section{Submit your manuscript to a SpringerOpen ${ }^{\odot}$ journal and benefit from:}

- Convenient online submission

- Rigorous peer review

- Immediate publication on acceptance

- Open access: articles freely available online

- High visibility within the field

- Retaining the copyright to your article

Submit your next manuscript at $\boldsymbol{\wedge}$ springeropen.com 